CASE REPORT

\title{
Alveolar haemorrhage in anti-glomerular basement membrane disease without detectable antibodies by conventional assays
}

\author{
D J Serisier, R C W Wong, J G Armstrong
}

Thorax 2006;61:636-639. doi: 10.1136/thx.2004.028985

\begin{abstract}
Anti-glomerular basement membrane (anti-GBM) disease represents the spectrum of disease attributable to circulating anti-GBM antibodies. While active anti-GBM disease in the absence of circulating anti-GBM antibodies has been described, it is considered rare with the use of current routinely available assays. We report four subjects with features consistent with active anti-GBM antibody disease without detectable antibodies by routinely available enzyme linked immunosorbent assay (ELISA) and immunoblot techniques. All were smokers who presented with diffuse alveolar haemorrhage, minimal renal involvement, and undetectable anti-GBM antibodies. Seronegative anti-GBM disease with predominant pulmonary involvement may be more common than previously appreciated and should be part of the differential diagnosis for otherwise unexplained diffuse alveolar haemorrhage. Renal biopsy with immunofluorescent studies should be considered in the diagnostic evaluation of such subjects, including those with idiopathic pulmonary haemosiderosis.
\end{abstract}

A nti-glomerular basement membrane (anti-GBM) disease is a rare disease with a reported incidence of approximately one per million. ${ }^{1}$ It represents the spectrum of disease attributable to circulating anti-GBM antibodies. The formation and deposition of these antibodies along glomerular and alveolar basement membranes is the pathological hallmark of the disease, with the alpha-3 chain of type IV collagen representing the antigenic target. ${ }^{2}$ Goodpasture's (pulmonary-renal) syndrome is the archetypal and most common presentation of this spectrum, characterised by rapidly progressive glomerulonephritis and pulmonary haemorrhage. The introduction of combination treatment with plasma exchange, cyclophosphamide, and prednisone in the 1970s has been associated with dramatic improvements in the prognosis of this condition.

Although apparently isolated pulmonary haemorrhage is recognised within the spectrum of anti-GBM disease, it occurs only occasionally and is almost universally associated with histological evidence of renal involvement. ${ }^{3}{ }^{4}$ Previous case reports have described patients with isolated alveolar haemorrhage subsequently shown to have anti-GBM disease. $^{5-7}$ Several of these patients had negative anti-GBM antibodies and were originally diagnosed with idiopathic pulmonary haemosiderosis. However, these older studies used indirect immunofluorescent (IIF) and radioimmunoassay (RIA) techniques. IIF techniques are generally considered to be less sensitive than currently available ELISA based assays due to difficulties in interpreting non-specific staining. ${ }^{2}$ We describe four subjects with active alveolar haemorrhage due to anti-GBM disease despite negative
anti-GBM antibodies by routinely available contemporary techniques.

\section{CASE REPORTS}

These cases represent four consecutive patients referred for respiratory opinion at two university teaching hospitals who were subsequently shown to have pulmonary manifestations of anti-GBM disease.

All subjects were current cigarette smokers of at least one packet per day and none had any occupational history of solvent or hydrocarbon exposure. None had any signs of systemic vasculitis or connective tissue disease, and all had negative anti-neutrophil cytoplasmic antibodies (ANCA). A summary of the investigations performed in all subjects is shown in table 1.

\section{Patient 1}

A 27 year old man presented with a l week history of haemoptysis. Urinalysis was unremarkable. Investigations showed normal renal function and haemoglobin. Chest radiography and computed tomographic (CT) scan showed diffuse airspace disease consistent with alveolar haemorrhage, and this was confirmed at bronchoscopy with progressively bloodier returns on sequential bronchoalveolar lavage. Anti-GBM antibodies by immunoblot (GBM Quickcard, Bio-Diagnostics, Upton upon Severn, UK) were undetectable. The patient had no further haemoptysis and his chest radiographic changes resolved. A presumptive diagnosis of idiopathic pulmonary haemosiderosis was made and on review 1 month later he remained well.

He failed to attend subsequent outpatient appointments and presented again nearly 2 years later with further diffuse alveolar haemorrhage. The chest radiograph again showed widespread alveolar opacification, he was anaemic and carbon monoxide gas transfer coefficient (KCO) was raised. Renal function and initial urinalysis were again unremarkable. Anti-GBM antibodies were now positive by both immunoblot (GBM Quickcard) and ELISA (EuroDiagnostica, Malmo, Sweden). He developed active urinary sediment without significant renal impairment and was commenced on plasmapheresis and immunosuppression with cyclophosphamide and methylprednisolone. He ceased smoking and 5 months later remained well on cyclophosphamide immunosuppression.

\section{Patient 2}

A 35 year old man presented with haemoptysis. Investigations revealed anaemia and normal renal function but active urinary sediment. A chest radiograph and high

Abbreviations: ANCA, anti-neutrophil cytoplasmic antibodies; GBM, glomerular basement membrane; IIF, indirect immunofluorescent; KCO, carbon monoxide transfer factor; RIA, radioimmunoassay 
Table 1 Summary of investigations performed, confirming alveolar haemorrhage and evaluating anti-GBM status

\begin{tabular}{|c|c|c|c|c|c|c|}
\hline & \multicolumn{2}{|l|}{ Subject 1} & \multirow{2}{*}{$\begin{array}{l}\text { Subject } 2 \\
\text { Episode } 1\end{array}$} & \multicolumn{2}{|l|}{ Subject $3^{*}$} & \multirow{2}{*}{$\frac{\text { Subject } 4^{*}}{\text { Episode } 2}$} \\
\hline & Episode 1 & Episode 2 & & Episode 2 & Episode 3 & \\
\hline Chest radiologyt & + & + & + & + & + & + \\
\hline Bronchoscopy $\ddagger$ & + & ND & ND & + & ND & + \\
\hline Kco (\% predicted, corrected for $\mathrm{Hb}$ ) & ND & $120 \%$ & $167 \%$ & $140 \%$ & $170 \%$ & $130 \%$ \\
\hline Urinalysis & - & - & $\begin{array}{l}\text { Prot trace } \\
\text { Blood 3+ }\end{array}$ & - & $\begin{array}{l}\text { Prot 1+ } \\
\text { Blood 1+ }\end{array}$ & - \\
\hline Haemoglobin (g/l) & 154 & 86 & 94 & 122 & 92 & 102 \\
\hline Serum creatinine $(\mathrm{mmol} / \mathrm{l})$ & 0.064 & 0.07 & 0.08 & 0.11 & 0.09 & 0.72 \\
\hline \multicolumn{7}{|l|}{ ANCA } \\
\hline IIF§ & - & Unint & - & - & - & - \\
\hline MPO/PR3 ELISA & ND & - & - & - & - & - \\
\hline \multicolumn{7}{|l|}{ Anti-GBM antibodies } \\
\hline Immunoblot (GBM QuickCard) & - & + & - & - & - & - \\
\hline ELISA (Immco Diagnostics) & & + & - & - & - & \\
\hline ELISA (Euro-Diagnostica) & & & - & & - & - \\
\hline IIF (The Binding Site) & & & - & & - & \\
\hline Renal biopsy** & ND & ND & + & ND & + & ND \\
\hline \multicolumn{7}{|c|}{$\begin{array}{l}\text { +, positive; -, negative; ND, not performed; Prot, protein; ELISA, enzyme linked immunosorbent assay; Kco, carbon monoxide transfer factor; GBM, glomerular } \\
\text { basement membrane; ANCA, anti-neutrophil cytoplasmic antibodies; IIF, indirect immunofluorescence; MPO, myeloperoxidase; PR3, proteinase-3; Unint, } \\
\text { uninterpretable. } \\
\text { Reference ranges for anti-GBM assays: Immunoblot = negative, ELISA }<20 \text { for both, IIF }<1: 2 \text {. } \\
\text { *Episode } 1 \text { for both subjects } 3 \text { and } 4 \text { predated involvement with the respiratory service (see text for details): anti-GBM antibodies at episode } 1 \text { were negative for } \\
\text { subject } 3 \text { (immunoblot) and positive for subject } 4 \text { (ELISA). } \\
\text { †Radiology positive if demonstrated bilateral alveolar opacification consistent with alveolar haemorrhage. } \\
\text { †Bronchoscopy positive if consistent with alveolar haemorrhage - that is, progressively bloodier returns on sequential bronchoalveolar lavage } \pm \text { numerous } \\
\text { haemosiderin laden macrophages seen on cytological examination. } \\
\text { \$lnova Slides (San Diego, USA) except subject } 4 \text { (performed on in-house cytospin slides). } \\
\text { TOrgentec (Mainz, Germany). }\end{array}$} \\
\hline
\end{tabular}

resolution CT scan of the chest showed widespread air space opacification consistent with diffuse alveolar haemorrhage, confirmed on respiratory function testing by substantially raised Kco. Anti-GBM antibodies by IIF (Monkey Kidney Slides, The Binding Site, Birmingham, UK), immunoblot (GBM Quickcard), and two different ELISA kits (EuroDiagnostica and Immco Diagnostics, Buffalo, NY, USA) were negative, but renal biopsy confirmed linear IgG deposition along the glomerular basement membrane with focal crescentic glomerulonephritis consistent with anti-GBM disease. He was commenced on plasmapheresis and immunosuppression with methylprednisolone and cyclophosphamide. Initially he deteriorated with progressive pulmonary haemorrhage, but subsequently improved with no further haemoptysis. He ceased smoking and at last follow up 12 months after completing 1 year of immunosuppressive therapy had no evidence of recurrent disease.

\section{Patient 3}

A 36 year old man presented initially with GBM positive renal failure and was treated with plasmapheresis, cyclophosphamide, and corticosteroids. He continued immunosuppressive therapy until he received a renal transplant after being antibody negative for over 12 months. After transplantation he remained well on cylosporine, azathioprine, and prednisone for 3 years and then cyclosporine and azathioprine for 6 years until he presented with haemoptysis 12 months before the current admission. Anti-GBM antibodies then were undetectable by immunoblot (GBM Quickcard) and his haemoptysis resolved spontaneously.

At the current episode he presented with haemoptysis, anaemia, and widespread bilateral alveolar infiltrates on the chest radiograph. He was receiving cyclosporine and azathioprine and had stable renal function with inactive urinary sediment. Respiratory function tests and bronchoscopic examination confirmed diffuse alveolar haemorrhage; however, anti-GBM antibodies were negative by immunoblot (GBM Quickcard) and ELISA (Immco Diagnostics). His haemoptysis and radiographic abnormalities resolved spontaneously.
He presented again 2 months later with further haemoptysis and radiographic evidence of diffuse alveolar haemorrhage, again confirmed on pulmonary function testing. Renal function remained stable but active urinary sediment was now present. Anti-GBM antibody tests were negative by IIF (The Binding Site), immunoblot (GBM Quickcard), and both ELISA assays (Immco Diagnostics and Euro-Diagnostica). Biopsy of his allograft demonstrated diffuse linear IgG on glomerular capillary walls and a diagnosis of recurrent antiGBM disease was made. Plasmapheresis and enhanced immunosuppression with cyclophosphamide and methylprednisolone were commenced with control of pulmonary haemorrhage. He ceased smoking and has had no further haemoptysis for 3 years.

\section{Patient 4}

A 17 year old man presented with acute renal failure and iron deficiency anaemia. Anti-GBM antibodies by ELISA were positive (Immco Diagnostics), with renal biopsy confirming linear IgG deposition along glomerular capillary walls. He was treated with plasmapheresis, cyclophosphamide, and prednisone and became anti-GBM antibody negative; however, his renal failure persisted and he commenced haemodialysis. Cyclophosphamide was stopped 2 months after commencement because of lymphopenia, and he continued treatment with prednisone alone.

Two months later he developed haemoptysis. The chest radiograph and CT scan showed airspace disease consistent with diffuse alveolar haemorrhage, and this was confirmed at bronchoscopy and on lung function testing. Anti-GBM antibodies were negative by both immunoblot (GBM Quickcard) and ELISA (Euro-Diagnostica) but a presumptive diagnosis of recurrent anti-GBM disease was made. Augmented immunosuppression with cyclophosphamide and methylprednisolone was commenced. He had no further haemoptysis and his chest radiographic abnormalities resolved. He ceased smoking and at last follow up 3 years later had had no further haemoptysis. 


\section{DISCUSSION}

The formation and deposition of anti-GBM antibodies along the glomerular and alveolar basement membranes is the pathological hallmark of anti-GBM disease, ${ }^{1}$ and there is compelling evidence that these antibodies are directly pathogenic. $^{89}$ The rarity of reported cases of anti-GBM disease without detectable antibodies supports this concept. Therefore, while the absence of detectable antibodies in the current cases may mitigate somewhat against this diagnosis, cases 1 and 2 had anti-GBM disease definitively proven by subsequent positive anti-GBM antibodies or renal biopsy with characteristic immunofluorescent findings. Furthermore, cases 3 and 4 had alveolar haemorrhage in the setting of previous GBM positive anti-GBM disease and exclusion of alternative diagnoses. These four cases raise interesting possibilities about the underlying pathophysiology of anti-GBM disease, and suggest that respiratory physicians should have a heightened awareness of this condition even in the absence of detectable antibodies by routinely available techniques.

It is possible that the prior administration of immunosuppressive therapy to subjects 3 and 4 may have influenced the results of antibody testing by reducing the levels of circulating anti-GBM antibodies below the detection limits of conventional routinely available assays. However, available evidence suggests that clinical manifestations of GBM disease reflect the pathogenic effects of the presence of antibodies irrespective of whether this is initial presentation or recurrence, ${ }^{8}$ although the actual antibody titre may not necessarily correlate to degree of activity. ${ }^{10}$ Consistent with this, the vast majority of reported cases of recurrent disease had recrudescence of detectable anti-GBM antibodies at clinical recurrence, despite prior immunosuppressive therapy. ${ }^{10-15}$ Furthermore, there is no evidence that prior immunosuppressive therapy alters the ability to detect antiGBM antibodies by changing the epitopes recognised by the antibodies or otherwise interfering with the assays used.

The inability to detect anti-GBM antibodies may reflect technical limitations of the routinely available assays (IIF, immunoblot and ELISA) rather than true antibody negativity. The fact that antibodies were detected in high titre in case 1 after initially being undetectable suggests that they may have been initially present in low levels. However, we used commercially available ELISA and immunoblot assays which are considered more sensitive than IIF. To ensure that the test results were not the result of technical error, all serum samples were retested on the corresponding assays shown in table 1 , and serum from patients 2 and 3 were sent to other laboratories for testing on alternative ELISA kits with persistently negative results. There is evidence that the diagnostic performance of different commercial ELISA kits varies, $^{16}$ although the four different anti-GBM assays (Immunoblot, two commercial ELISA kits, and IIF using monkey kidney slides; table 1) used to assess our cases were found to have equal sensitivity in seven patients with renal biopsy proven anti-GBM disease (Wilson RJ et al, unpublished data). However, the possibility remains that all the assays used are relatively insensitive for very low levels of circulating anti-GBM antibodies.

Salama et $a l^{17}$ have estimated that seronegative anti-GBM disease may occur in $2-3 \%$ of patients based on their experience as a reference laboratory for anti-GBM antibody testing in the UK. They recently described three patients with negative antibodies by ELISA but typical renal biopsy changes of anti-GBM disease, ${ }^{10}$ and were subsequently able to detect circulating antibodies in two of these patients using a new highly sensitive biosensor assay system. The authors had initially speculated that these patients would have low circulating levels of antibodies detectable only by the more sensitive test, but the levels were actually shown to be similar to those seen in the lower range in other patients. Unfortunately there was insufficient serum left to perform this more sensitive assay in our patients, which may have provided useful information about very low levels of circulating anti-GBM antibodies.

A review of 10 reports describing apparently isolated alveolar haemorrhage subsequently shown to represent anti-GBM disease ${ }^{4-7}{ }^{18-23}$ found that only six of 13 cases were initially positive for serum anti-GBM antibodies. This lends support to the possibility that seronegative anti-GBM disease by routine anti-GBM assays may explain isolated alveolar haemorrhage more often than is currently appreciated.

Furthermore, our series raises the intriguing possibility that idiopathic pulmonary haemosiderosis may represent a "form fruste" of anti-GBM disease. Case 1 was initially given this diagnosis of exclusion before anti-GBM antibodies became positive. Others have reported similar patients with isolated pulmonary haemorrhage and no evidence of antiGBM disease diagnosed initially as idiopathic pulmonary haemosiderosis who subsequently developed renal involvement with positive anti-GBM antibodies. ${ }^{415}$ Donald et $a l^{23}$ actually described "idiopathic pulmonary haemosiderosis" in a small series of subjects with isolated pulmonary haemorrhage and negative antibodies, despite one subject having linear IgG staining of glomerular capillary loops on renal biopsy.

It may be that subjects with very low levels of anti-GBM antibodies develop isolated pulmonary haemorrhage when exposed to the right precipitant. In anti-GBM disease, development of pulmonary involvement requires an additional non-specific lung injury that increases alveolarcapillary permeability. Concurrent infection, fluid overload, high concentrations of inspired oxygen, volatile hydrocarbons, and cigarette smoking have all been implicated. ${ }^{24}$ Cigarette smoking is strongly correlated with the development of pulmonary haemorrhage in patients with anti-GBM disease. ${ }^{25}$ Our subjects were all smokers who presented with alveolar haemorrhage and minimal evidence of active renal involvement. Such subjects may represent a subgroup with "early" anti-GBM disease and relatively low circulating antibodies that manifest pulmonary disease due to the direct effects of cigarette smoke on the alveolar walls. It is possible that smoking may even stimulate production of anti-GBM antibodies by allowing direct contact between the alveolar basement membrane and the immune system.

Our series suggests that alveolar haemorrhage in anti-GBM disease that is seronegative by routinely available assays may be more common than previously appreciated and clinicians should have an enhanced awareness of this possibility, especially in the setting of recurrent disease. This is particularly important for respiratory physicians who manage subjects with apparently isolated diffuse alveolar haemorrhage. A more aggressive approach to the investigation of "idiopathic pulmonary haemosiderosis" may be warranted; evidence of even minimal renal involvement should point to a strong consideration of renal biopsy. Early diagnosis of anti-GBM disease in such patients would mandate more vigilant monitoring for development of renal disease and strengthen the argument for smoking cessation.

\section{Authors' affiliations}

D J Serisier, Department of Respiratory Medicine, Mater Adult Hospital, South Brisbane, Queensland 4101, Australia

R C W Wong, Queensland Health Pathology Service, Princess Alexandra Hospital, Woolloongabba, Queensland 4102, Australia J G Armstrong, Respiratory Medicine Department, Princess Alexandra Hospital, Woolloongabba, Queensland 4102, Australia 
Correspondence to: Dr D J Serisier, Department of Respiratory Medicine, Mater Adult Hospital, Raymond Tce, South Brisbane, Queensland 4101, Australia; david_serisier@mater.org.au

Received 21 May 2004

Accepted 8 October 2004

\section{REFERENCES}

1 Savage C, Pusey C, Bowman C, et al. Antiglomerular basement membrane antibody mediated disease in the British Isles 1980-4. BMJ 1986;292:301-4.

2 Hellmark T, Segelmark M, Bygren P, et al. Glomerular basement membrane antibodies. In: Peter JB, Shoenfeld Y, eds. Autoantibodies. 1 st ed. Amsterdam: Elsevier, 1996:291-8.

3 Pusey CD. Anti-glomerular basement membrane disease. Kidney Int 2003;64:1535-50.

4 Rosenblum ND, Colvin RB. A 13 year old girl with gross haematuria 4 years after a diagnosis of idiopathic pulmonary haemosiderosis. N Engl J Med 1993;328:1183-90.

5 Mathew TH, Hobbs JB, Kalowski S, et al. Goodpasture's syndrome: normal renal diagnostic findings. Ann Intern Med 1975;82:215-8.

6 Carre PH, Lloveras JJ, Didier A, et al. Goodpasture's syndrome with normal renal function. Eur Respir J 1989;2:911-5.

7 Zimmerman SW, Varanasi UR, Hoff B. Goodpasture's syndrome with normal renal function. Am J Med 1979;66:163-71.

8 Wilson C, Dixon J. Anti-glomerular basement membrane antibody-induced glomerulonephritis. Kidney Int 1973:3:74-89.

9 Lerner R, Glassock R, Dixon F. The role of antiglomerular basement antibody in the pathogenesis of human glomerulonephritis. J Exp Med 1967; 126:989-1004

10 Rees AJ, Lockwood CM, Peters DK. Enhanced allergic tissue injury in Goodpasture's syndrome by intercurrent bacterial infection. BMU 1977;2:723-6.

11 Deodhar HA, Marshall RJ, Sivathondan Y, et al. Recurrence of Goodpasture's syndrome associated with mesangiocapillary glomerulonephritis. Nephrol Dial Transplant 1994;9:72-5.
12 Klasa RJ, Aboud RT, Ballon HS, et al. Goodpasture's syndrome: recurrence after a five-year remission. Am J Med 1988;84:751-5.

13 Trpkov K, Abdulkareem F, Jim K, et al. Recurrence of anti-GBM disease twelve years after transplantation associated with de novo IgA nephropathy. Clin Nephrol 1998;49:124-8.

14 Fonck C, Lout G, Cosyns J, et al. Recurrent fulminant anti-glomerular basement membrane nephritis at a 7-year interval. Am J Kidney Dis 1998:32:323-7.

15 Almkuist RD, Buckalew VM, Hirszel P, et al. Recurrence of anti-glomerular basement membrane antibody mediated glomerulonephritis in an isograft. Clin Immunol Immunopathol 1981;18:54-60.

16 Jaskowski TD, Martins TB, Litwin CM, et al. Comparison of four enzyme immunoassays for the detection of immunoglobulin $\mathrm{G}$ antibody against glomerular basement membrane. J Clin Lab Anal 2002;16:143-5.

17 Salama AD, Dougan T, Levy JB, et al. Goodpasture's disease in the absence of circulating anti-glomerular basement membrane antibodies as detected by standard techniques. Am J Kidney Dis 2002;39:1162-7.

18 Wilson CB, Smith RC. Goodpasture's syndrome associated with influenza A2 virus infection. Ann Intern Med 1972;76:91-4.

19 Tobler A, Schurch E, Altermatt HJ, et al. Anti-basement membrane antibody disease with severe pulmonary haemorrhage and normal renal function. Thorax 1991;46:68-9.

20 Bell DD, Moffat SL, Singer M, et al. Antibasement membrane disease without clinical evidence of renal disease. Am Rev Respir Dis 1990;142:234-7.

21 Bailey RR, Simpson IJ, Lynn KL, et al. Goodpasture's syndrome with normal renal function. Clin Nephrol 1981;15:211-5.

22 Katz SM, Foster E, Miller AS, et al. Goodpasture's syndrome mimicking idiopathic pulmonary haemosiderosis. Ann Clin Lab Sci 1989;19:280-6.

23 Donald KJ, Edwards RL, McEvoy JDS. Alveolar capillary basement membrane lesions in Goodpasture's syndrome and idiopathic pulmonary haemosiderosis. Am J Med 1975;59:642-9.

24 Cameron JS. Glomerulonephritis in renal transplants. Transplantation 1982;34:237-44.

25 Donaghy M, Rees A. Cigarette smoking and lung haemorrhage in glomerulonephritis caused by autoantibodies to glomerular basement membrane. Lancet 1983;2:1390-3.

\section{LUNG ALERT}

\section{No mortality benefit seen with methylprednisolone in ARDS}

\ Steinberg KP, Hudson LD, Goodman RB, et al. The National Heart, Lung, and Blood Institute Acute Respiratory Distress Syndrome (ARDS) Clinical Trials Network. Efficacy and safety of corticosteroids for persistent acute respiratory distress syndrome. N Engl J Med 2006;354:1671-84

7 he use of corticosteroids in acute respiratory distress syndrome (ARDS) is controversial, with previous studies showing no benefit of high dose steroids in early ARDS but indicating a possible role for moderate doses in late ARDS ( $>7$ days after its onset). This multicentre double blind trial randomised 180 intubated and mechanically ventilated patients 7-28 days after the onset of ARDS to receive either intravenous methlyprednisolone or placebo. The primary outcome was mortality at 60 days, with secondary outcomes including ventilator-free days, days without organ failure, and infectious complications at 28 days.

There was no difference in mortality between the treatment and placebo groups at 60 days $(29.2 \% \vee 28.6 \%)$ or 180 days $(31.5 \% \vee 31.9 \%)$. If the patients had ARDS for longer than 14 days before enrolment, methlyprednisolone was associated with increased mortality at 60 days $(35 \% v 8 \% ; \mathrm{p}=0.02)$. At 28 days the treatment group had more ventilator-free days $(11.2 \vee 6.8 ; \mathrm{p}<0.001)$, more ICU-free days $(8.9 \vee 6.2 ; \mathrm{p}<0.02)$, and fewer episodes of shock $(6 v 17 ; \mathrm{p}=003)$, but they were more likely to have to resume assisted ventilation $(20 v 6$; $\mathrm{p}=0.008)$. They also had higher glucose levels and more episodes of severe neuromyopathy ( $9 v 0 ; \mathrm{p}=0.001)$, although there was no difference in the rate of infectious complications.

From this evidence, it appears that the use of methlyprednisolone in ARDS confers no survival benefit and may be harmful if initiated late. However, the reasons why initial improvements in cardiovascular and respiratory parameters are not translated into improved survival are unclear, and a better understanding of the mechanisms of ARDS may help to define an optimal time frame and regimen for corticosteroids in the disease.

C Snelson

Specialist Registrar, George Eliot Hospital, Nuneaton, Warwickshire, UK; catherinesnelson@hotmail.com 